\title{
Universality of (2+1)-dimensional restricted solid-on-solid models
}

\author{
Jeffrey Kelling, ${ }^{1,2}$ Géza Ódor, ${ }^{3}$ and Sibylle Gemming ${ }^{2,4}$ \\ ${ }^{1}$ Department of Information Services and Computing, Helmholtz-Zentrum Dresden-Rossendorf, P. O. Box 5101 19, 01314 Dresden, Germany \\ ${ }^{2}$ Institute of Ion Beam Physics and Materials Research, Helmholtz-Zentrum Dresden-Rossendorf, P. O. Box 5101 19, \\ 01314 Dresden, Germany \\ ${ }^{3}$ Institute of Technical Physics and Materials Science, Centre for Energy Research of the Hungarian Academy of Sciences, \\ P. O. Box 49, H-1525 Budapest, Hungary \\ ${ }^{4}$ Institute of Physics, TU Chemnitz, 09107 Chemnitz, Germany
}

(Received 13 May 2016; published 5 August 2016)

\begin{abstract}
Extensive dynamical simulations of restricted solid-on-solid models in $D=2+1$ dimensions have been done using parallel multisurface algorithms implemented on graphics cards. Numerical evidence is presented that these models exhibit Kardar-Parisi-Zhang surface growth scaling, irrespective of the step heights $N$. We show that by increasing $N$ the corrections to scaling increase, thus smaller step-sized models describe better the asymptotic, long-wave-scaling behavior.
\end{abstract}

DOI: 10.1103/PhysRevE.94.022107

\section{INTRODUCTION}

The Kardar-Parisi-Zhang (KPZ) equation [1] describes the evolution of a fundamental, nonequilibrium surface growth model by a Langevin equation,

$$
\partial_{t} h(\mathbf{x}, t)=\sigma \nabla^{2} h(\mathbf{x}, t)+\lambda[\nabla h(\mathbf{x}, t)]^{2}+\eta(\mathbf{x}, t) .
$$

The scalar field $h(\mathbf{x}, t)$ is the height, progressing in the $D$-dimensional space relative to its mean position, that moves linearly with time $t$. A smoothing surface tension is represented by the coefficient $\sigma$, which competes a curvature-driven propagation, described by the nonlinear coefficient $\lambda$ and a zeroaverage Gaussian stochastic noise. This noise field exhibits the variance $\left\langle\eta(\mathbf{x}, t) \eta\left(\mathbf{x}^{\prime}, t^{\prime}\right)\right\rangle=2 \Gamma \delta^{D}\left(\mathbf{x}-\mathbf{x}^{\prime}\right)\left(t-t^{\prime}\right)$, with an amplitude related to the temperature in the equilibrium system, and \langle\rangle denotes a distribution average. Besides describing the dynamics of simple growth processes [2], KPZ was inspired in part by the stochastic Burgers equation [3] and is applicable for randomly stirred fluids [4], for directed polymers in random media [5], for dissipative transport [6,7], and for the magnetic flux lines in superconductors [8].

Discretized versions have been studied frequently over the past few decades [9-11]. The morphology of a surface of linear size $L$ can be described by the squared interface width

$$
W^{2}(L, t)=\frac{1}{L^{2}} \sum_{i, j}^{L} h_{i, j}^{2}(t)-\left[\frac{1}{L^{2}} \sum_{i, j}^{L} h_{i, j}(t)\right]^{2} .
$$

In the absence of any characteristic length, simple growth processes are expected to be scale invariant,

$$
W(L, t) \propto L^{\alpha} f\left(t / L^{z}\right),
$$

with the universal scaling function $f(u)$

$$
f(u) \propto\left\{\begin{array}{lll}
u^{\beta} & \text { if } & u \ll 1 \\
\text { const. } & \text { if } \quad u \gg 1
\end{array}\right.
$$

Here $\alpha$ is the roughness exponent in the stationary regime, when the correlation length has exceeded $L$ and $\beta$ is the growth exponent, describing the intermediate time behavior. The dynamical exponent $z$ can be expressed as the ratio of the growth exponents

$$
z=\alpha / \beta
$$

and, due to the Galilean invariance, the $\alpha+z=2$ relation holds as well.

While in $D=1+1$ exact solutions are known, due to the Galilean symmetry [4] and an incidental fluctuationdissipation symmetry [12], in higher dimensions KPZ has been investigated by various analytical [13-18] and numerical methods [19-22], but still debated issues remain. For example, there is a controversy on the surface growth exponents of the $D=2+1 \mathrm{KPZ}$, obtained by recent simulations $[23,24]$ and a field-theoretical study [25]. Assuming that the height correlations do not exhibit multiscaling and satisfy an operator product expansion Ref. [25] concluded that growth exponents are rational numbers in two and three dimensions [25]. This was in accordance with some earlier restricted solidon-solid (RSOS) model simulation results [26,27]. Recent high precision simulations $[23,24,28-30]$ all excluded this and concluded $\alpha=0.393$ (4) [23,24,30] and $\beta=0.2414$ (15) [23]. RSOS models are defined by deposition at random sites if the local height difference satisfies

$$
\left|h(\mathbf{x}, t)-h\left(\mathbf{x}^{\prime}, t\right)\right| \leqslant N .
$$

Very recently, Kim [31] investigated RSOS models with maximum step sizes $N=1,2, \ldots, 7$. As he increased $N$, the roughness exponent $\alpha$ seemed to converge to $4 / 10$ and the growth exponent $\beta$ to $1 / 4$, in agreement with Refs. [25-27]. This issue is important, because one may speculate that discretized simulations cannot describe the local singularities of continuum models, i.e., finite slopes may cause corrections, responsible for the longstanding debate between field theory and discrete model simulations.

In this paper we show that the converse is true. By performing very careful corrections-to-scaling analysis on the model of Refs. [27,31], we show that even in case of $N>1$ the rational numbers of Refs. [25-27] can be excluded in the $L \rightarrow \infty$ limit. Local slopes analysis shows that the $N=1$ case has the smallest corrections and describes the KPZ universality scaling the best. For $N>1$ corrections corresponding shorter wavelengths are introduced. Our findings are in full agreement 
with the scaling results obtained for ballistic growth models [24,32,33].

\section{MODELS AND SIMULATION ALGORITHMS}

In order to enable long-time surface growth simulations of large systems, a multisurface-like parallel implementation of the RSOS model has been created for graphics processing units (GPUs). Two parallelization approaches have been combined as follows.

Since GPUs feature a number of vector processors, multiples of 128 realizations of the model were simulated simultaneously. This creates a data-parallel workload, which can straightforwardly be vectorized. Each single instruction multiple thread (SIMT) unit of the GPU updates 128 realizations, in which the sequence of randomly selected coordinates for update is the same. This correlation was broken by updating only half of the selected lattice sites in each attempt. If more realizations were simulated, different sets of 128 realizations evolved completely independently.

In order to handle large systems effectively, a domain decomposition (DD) was also used to distribute the work of realizations among multiple SIMT elements. A double-tiling scheme was applied by splitting up the simulation cells into tiles, split further into two subtiles along each spatial direction [34]. In the present two-dimensional problem this yields $2^{d}=$ 4 sets of subtiles, each of which can be updated by multiple independent workers. After each lattice sweep the origin of the DD was moved randomly to eliminate correlations. Implementation details will be published elsewhere [35].

Roughening of $(2+1)$-dimensional RSOS surfaces was studied for restriction parameters $N=1,3,5,7$, by starting from flat initial conditions. To obtain estimates for the exponent $\beta$, the growth of surfaces was followed up to $t=10^{5}$ Monte Carlo steps (MCS), which is well before the correlation length approaches the system sizes: $L=$ 4096, 8192, and 9605 studied here (throughout this paper the time is measured in MCS). The largest system size was bounded by memory constraints, filling up $12 \mathrm{~GB}$ of the NVIDIA K40 GPU, and leaving some memory for the random number generator (RNG) states. The results were averaged over $n=768,128$, and 128 realizations, respectively, where the latter two correspond to only one multisurface run.

The exponent $\alpha$ was determined by a finite-size scaling analysis of the saturation roughness of system sizes between $L=64$ and $L=512$. To keep the noise amplitude, constant we used domain sizes of $8 \times 8$ lattice sites. We determined the interface width by averaging over $W(L, t)$ for times $t \geqslant$ $t_{\text {start }}$ and for all samples. We checked whether the averaged values belong to the steady state $t>t_{\text {steady* }}$ by varying $t_{\text {start }}$, the onset times of the measurements. We estimated $t_{\text {steady* }}$ via the relation

$$
a_{N} L^{\alpha}=b_{N} t_{\text {steady }}^{\beta},
$$

using the parameters $a_{N}$ and $b_{N}$, deduced from fitting in small systems.

In order to estimate the asymptotic values of $\alpha$ and $\beta$ for $L \rightarrow \infty$ and $t \rightarrow \infty$, respectively, a local slopes analysis of the scaling laws was performed [36]. We calculated the effective exponents

$\alpha_{\mathrm{eff}}\left(\frac{L-L / 2}{2}\right)=\frac{\ln W(L, t \rightarrow \infty)-\ln W(L / 2, t \rightarrow \infty)}{\ln (L)-\ln (L / 2)}$,

$\beta_{\mathrm{eff}}\left(\frac{t_{i}-t_{i / 2}}{2}\right)=\frac{\ln W\left(L \rightarrow \infty, t_{i}\right)-\ln W\left(L \rightarrow \infty, t_{i / 2}\right)}{\ln \left(t_{i}\right)-\ln \left(t_{i / 2}\right)}$.

In our studies the simulation time between two measurements is increased exponentially,

$$
t_{i+1}=\left(t_{i}+10\right) \mathrm{e}^{m}
$$

using $m=0.01$ and $t_{0}=0$, while statistical uncertainties are provided as $1 \sigma$ standard errors, defined as $\Delta_{1 \sigma} x=$ $\sqrt{\left\langle x^{2}\right\rangle-\langle x\rangle^{2}} /(N-1)$.

\section{SURFACE GROWTH RESULTS}

\section{A. The growth regime}

The growth of the surface roughness follows apparently the same, clear, power law for all considered $N$ [Fig. 1(a)]. The local slopes plots [Fig. 1(b)], using (9), show an effective growth exponent $\beta_{\text {eff }} \approx 0.25$ for $N=5,7$ for $t \leqslant 1000 \mathrm{MCS}$ $\left(t^{-1 / 4} \approx 0.18\right)$, in agreement with Kim's results [31]. Later, the effective growth exponent decreases for all $N>1$, followed over two orders of magnitude in time in Fig. 1.

Expecting independence of $\beta$ from $N$, it follows that the asymptotic estimates $\beta_{N}$ should be the same. By assuming power-law corrections to the asymptotic scaling $W(L \rightarrow$ $\infty, t) \propto t^{\beta}\left(1+t^{-x}\right)$, we obtained a minimal variance of the $\beta_{N>1}$ estimates in case of $x \simeq 0.25$. Therefore, we plotted our $\beta_{\text {eff }}$ results on the $\sim 1 / \sqrt[4]{t}$ scales, which makes the tails of the curves straight in the $N \rightarrow \infty$ limit. Logarithmic corrections to scaling were also tested, but they did not improve the extrapolations.

Table I lists the obtained estimates for $\beta$ for the considered system sizes. Results for different $N>1$ are practically identical and are thus averaged to give a common value. The case $N=1$ is listed separately, due to the different corrections to scaling. For $N=1, \beta_{\text {eff }}$ can be best extrapolated by a power-law fit with $x=0.90(2)$. This is in good agreement with the results of Ref. [37], where $x \simeq 0.96 \simeq 4 \beta$ is reported, based on the KPZ ansatz hypothesis. This motivated us testing more general scaling forms, with correction exponents multiple of $x=\beta$. When we combined the effective exponent forms of $N=1$ and $N>1$,

$$
\beta_{\text {eff }}(1 / t)=\beta+a_{1} / t^{4 \beta}+a_{2} / t^{\beta},
$$

with free parameters $a_{i}$, fitting for $t \geqslant 148$ MCS resulted in good agreement for most of the growth region. This is shown for $L=8192$ by the dashed lines in Fig. 1(b). From these extrapolations we obtained the estimates: $\beta_{N>1}=0.2395(5)$ and $\beta_{1}=0.2415(5)$.

As we can observe in Fig. 1, the effective exponents suffer from stronger corrections for $N>1$ than in the $N=1$ case. Furthermore, our data suggest a possible oscillating convergence of $\beta_{\text {eff }}$ for $N>1$, as reported in simulations 

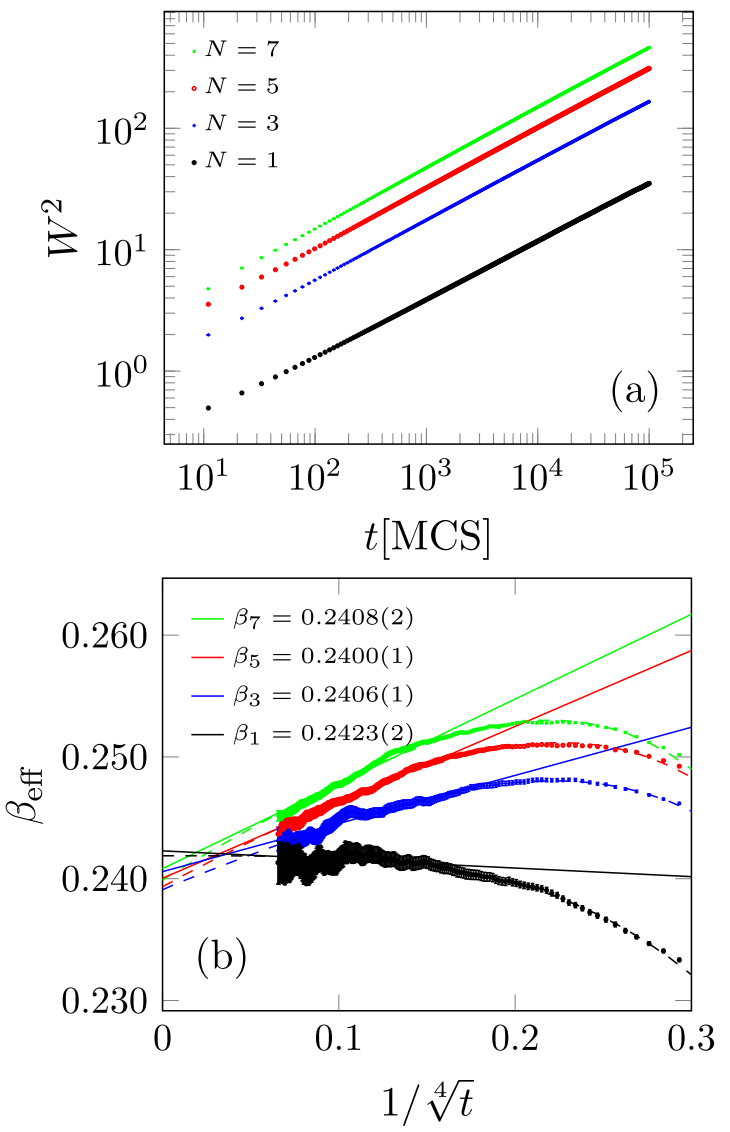

FIG. 1. (a) Squared roughness $\left(W^{2}\right)$ of surfaces of size $V=$ $4096^{2}$ (256 realizations) in the scaling regime (error bars are smaller than symbols). (b) Local slopes analysis of roughness scaling for size $V=8192^{2}$ (128 realizations). Straight lines are linear fits to the tail $(t \geqslant 1260 \mathrm{MCS})$, extrapolating to $t \rightarrow \infty$, assuming $\sqrt[4]{t}$ corrections. Uncertainties given for $\beta_{N}$ are errors of the singular linear fits displayed in the plot. The black dashed line is the PL extrapolation for $N=1$. The dashed lines corresponding in color to the respective plots for $N>1$ are fits of the form (11). All PL fits were performed for $t \geqslant 148 \mathrm{MCS}$. Both figures show $N=1,3,5,7$ (bottom to top).

of the ballistic deposition model (BD) [24]. Extrapolations based on the form (11), while in good agreement within the observed region, are prone to overfitting, where they cannot cover all possible corrections. The values for $\beta_{N>1}$ are thus underestimated if the effective exponents do indeed show oscillating convergence.

The estimates show no clear dependence on system size, and thus it can be safely assumed that all simulations are well within the scaling regime and do not suffer from finite-size

TABLE I. Extrapolated $\beta$ results for different $N$. For $N=1$ figures in the parentheses are fit errors from PL extrapolations. For $N>1$, given margins are $1 \sigma$ standard errors from averaging over $N=3,5$, and 7 .

\begin{tabular}{lccc}
\hline \hline$L$ & 4096 & 8192 & 9605 \\
\hline$\beta_{1}$ & $0.2412(1)$ & $0.2418(1)$ & $0.2415(1)$ \\
$\beta_{N>1}$ & $0.2404(3)$ & $0.2405(3)$ & $0.2410(3)$ \\
\hline \hline
\end{tabular}

effects. All results are within the margin of error of the octahedron model $\beta=0.2415$ (15) [23]. Most notably this is also the case for the estimates for $N>1$. Statistical error measures for single extrapolations do not account for systematic contributions such as from the choice of the extrapolation form or the interval used for a fit. This can be clearly seen by the fact that many extrapolated values listed above do not agree with each other within such a margin. The spread of these different estimates itself provides a more useful estimate of the margin of error. Overall, the presented data support $\beta=0.241(1)$.

Since the curves in Fig. 1 correspond to the same $L$ and sample size $n$, one can observe that the signal-to-noise ratio $(S / N)$, the ratio between the interface width and the sample variance, increases with $N$. For $N=7$ this is higher by a factor of $\sim 3.6$, while for $N=3$ the $S / N$ is about $\sim 2.5$ bigger than that of the $N=1$ result. Presumably, the decrease of relative noise level is the consequence of a kind of self-averaging, since systems with larger allowed $N$ accommodate more surface information than smaller ones. It is tempting to exploit this property by choosing larger height differences in the simulations, even if this can be implemented less efficiently.

\section{B. The steady state}

Direct fitting of the finite-size scaling form

$$
W_{\text {sat }}(L) \sim L^{\alpha},
$$

for $32 \leqslant L \leqslant 512$ and $t_{\text {start }}=50 t_{\text {steady* }}$ yields the following estimates:

$$
\alpha_{\text {fit }}=\left\{\begin{array}{lll}
0.392(1) & 0.392(5) & N=1 \\
0.401(2) & 0.400(4) & N=3 \\
0.402(2) & 0.401(4) & N=5 \\
0.402(2) & & N=7
\end{array} .\right.
$$

For comparison, Kim's results [31] are shown in the second column. When we decrease $t_{\text {start }}$ our values decrease slightly but fall inside the error margins if $t_{\text {start }} \geqslant 2 t_{\text {steady* }}$. So, direct fits match perfectly those of Ref. [31], obtained by sequential Monte Carlo updates.

However, if the $L=32$ data are excluded, our estimates become significantly lower, warning for strong corrections to scaling. This can also be seen with the help of the effective exponents in Fig. 2 calculated by (8). There is a clear tendency for $\alpha_{\text {eff }}$ to decrease as we increase the system size for the $N>1$ cases. The approach to $L \rightarrow \infty$ is nonlinear, but the number of points is insufficient for power-law (PL) extrapolations to produce consistent estimates. We plotted the $\alpha_{\text {eff }}(L)$ results on the $1 / \sqrt{L}$ scale, resulting in points that can be settled on straight lines. Linear extrapolation to asymptotically large sizes yields:

$$
\alpha= \begin{cases}0.391(1) & N=1 \\ 0.386(1) & N>1\end{cases}
$$

Corrections to finite-size scaling (12) in case of $N=1$ are small, explaining the good agreement between local slopes analysis and the direct fit. The slight difference between the results for $N=1$ and $N>1$ may be attributed to the fact that our data points are not from deep enough in the steady state. This might also explain the disagreement with the results of a 

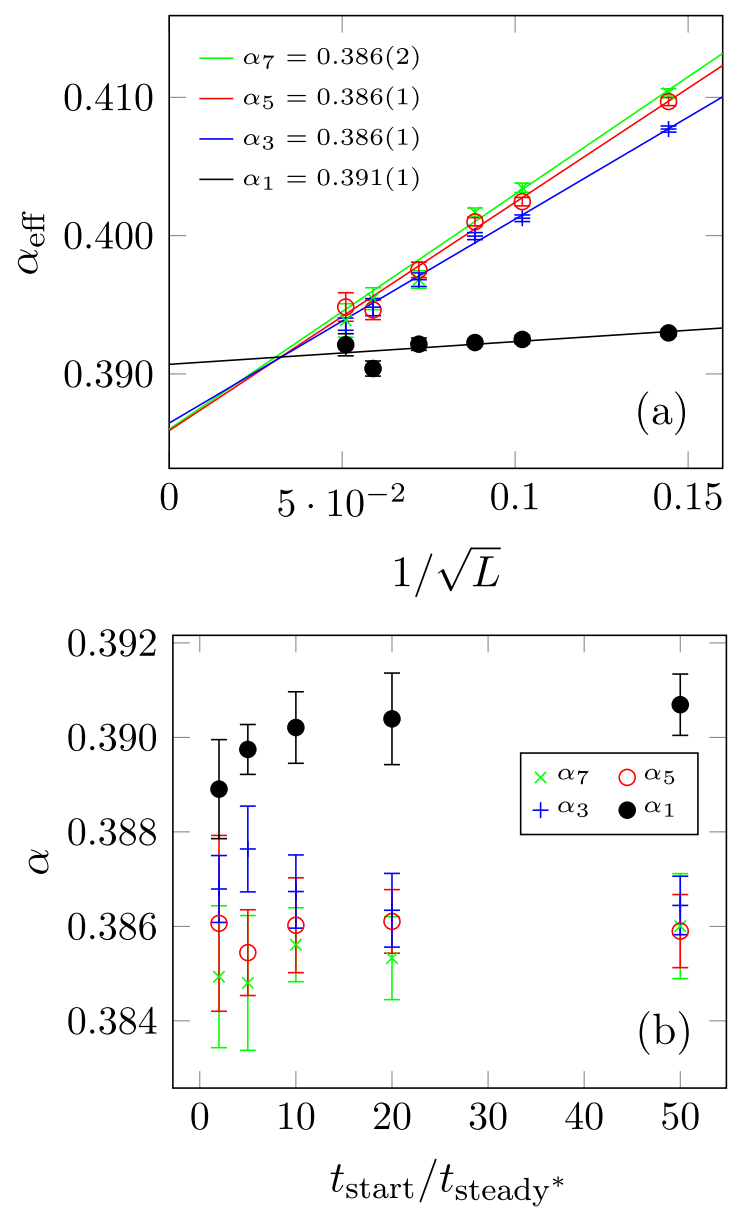

FIG. 2. (a) Local slopes of finite-size scaling analysis with $N=$ $1,3,5,7$. Error bars are propagated $1 \sigma$ errors. Straight lines are linear fits to extrapolate to infinity, uncertainties given for $\alpha_{N}$ are pure fit errors. Steady-state data taken for $t>t_{\text {start }}=50 t_{\text {steady* }}$ (see text). (b) Dependence of extrapolated $\alpha$ on $t_{\text {start }}$ is weak. Both figures: Sample sizes are at least $1024-2048$ realizations and $\geqslant 8192$ realizations for $L \leqslant 64$. All system sizes taken into account for finite-size scaling are listed in Fig. 3, where the considered time scales can also be read off.

recent study [30], which reported $\alpha=0.3869$ (4) for $N=1$. There is a further uncertainty of the extrapolation to $L \rightarrow$ $\infty$, which is not accounted for by the fit errors. With the assumption of an intrinsic width: $W_{i}^{2}=0.2$ [32], the local slopes analysis shows stronger corrections to scaling, therefore we did not apply this in our study.

The observation of stronger corrections for larger $\mathrm{Ns}$ is consistent with a recent analysis of the BD [24]. This study found that corrections to scaling, for both $\alpha$ and $\beta$, are reduced, when the BD surface is smoothened by binning of the surface positions before analysis, thereby decreasing the height differences between neighboring sites. Binning of the surface did not change the universal behavior; it only decreased nonuniversal corrections. The corrections produced even an oscillatory approach to the asymptotic values of the exponents. This can explain why our simple extrapolations of $\alpha_{\text {eff }}$ (Fig. 2) and $\beta_{\text {eff }}$ (Fig. 1) for $N>1$ undershoot those of $N=1$.

All of our estimates up to $N \leqslant 7$, obtained by the local slopes analysis, are in the range $\alpha=0.390(4)$, which clearly
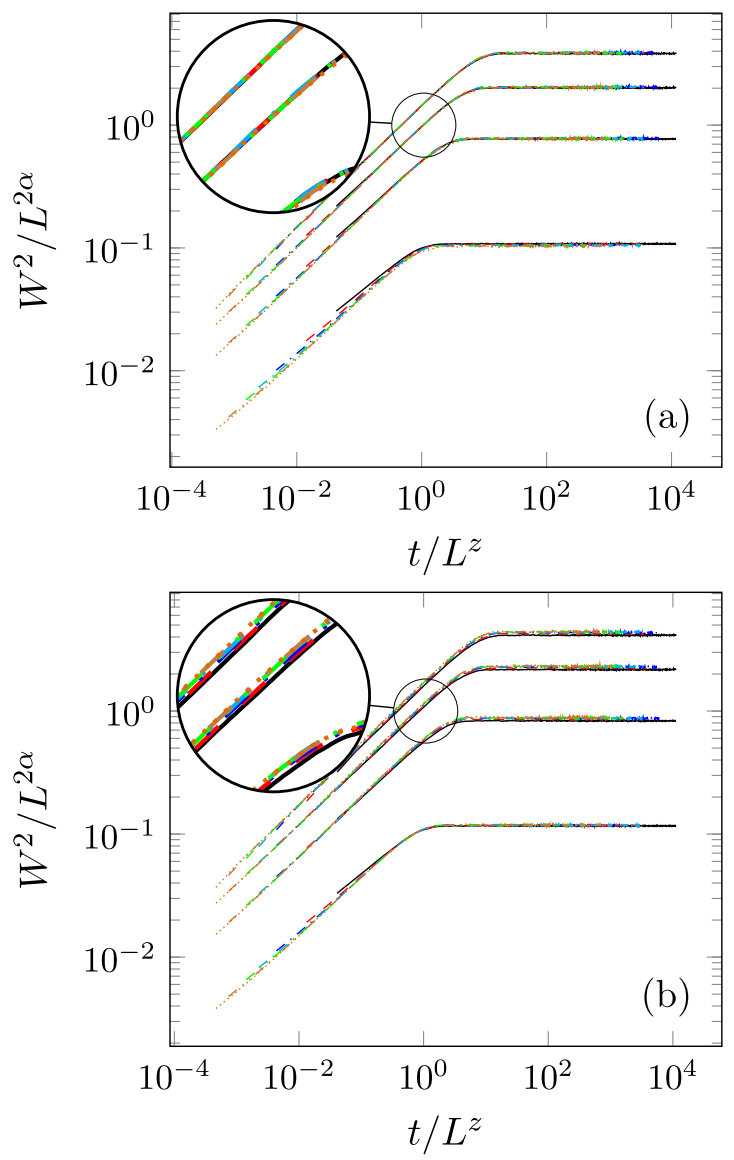

FIG. 3. Collapse of squared roughness in the steady state for $N=1,3,5,7$ (from bottom to top). Panel (a) shows a perfect collapse for $N>1$, using $\alpha=0.4$ and $\beta=0.25(z=\alpha / \beta=1.6)$. Panel (b) shows a collapse using $\alpha=0.389$ and $\beta=0.241(z \approx 1.61)$. This looks perfect for $N=1$ but not for $N>1$.

excludes $\alpha=2 / 5$. Plugging our $\alpha$ and $\beta$ results into the scaling relation (5) we get the dynamical exponent estimates $z_{N=1}=1.61(2)$ and $z_{N>1}=1.60(2)$, respectively. The scaling law following from the Galilean invariance is satisfied with these exponents both for $N=1: \alpha+z=2.01(2)$ and $N>1$ : $\alpha+z=1.99(2)$ within error margins.

We have also tested the scaling form (3) numerically by using our $\alpha$ and $\beta$ values. As Fig. 3 shows, good data collapses can be obtained for $N>1$ and even a perfectly looking one for $N=1$. For $N>1$ in the growth regime a perfect one can also be achieved assuming the values suggested by Kim and Kosterlitz [26] [Fig. 3(a)]. This can be understood by taking into account the corrections to scaling we explored above. Effective exponents for early times and small systems agree with the conjecture by Ref. [26] and indeed the most strongly outlying curves in Fig. 3(a), correspond to smaller systems.

Moments of the width and height distributions are defined as:

$$
\Phi_{L}^{n}\left[\varphi_{L}\right]=\int_{0}^{\infty}\left(\varphi_{L}-\left\langle\varphi_{L}\right\rangle\right)^{n} P_{L}\left(\varphi_{L}\right) d \varphi_{L},
$$

where $P_{L}\left(\varphi_{L}\right)$ denotes the probability distribution corresponding to the interface observable $\varphi_{L}$. We calculated some 

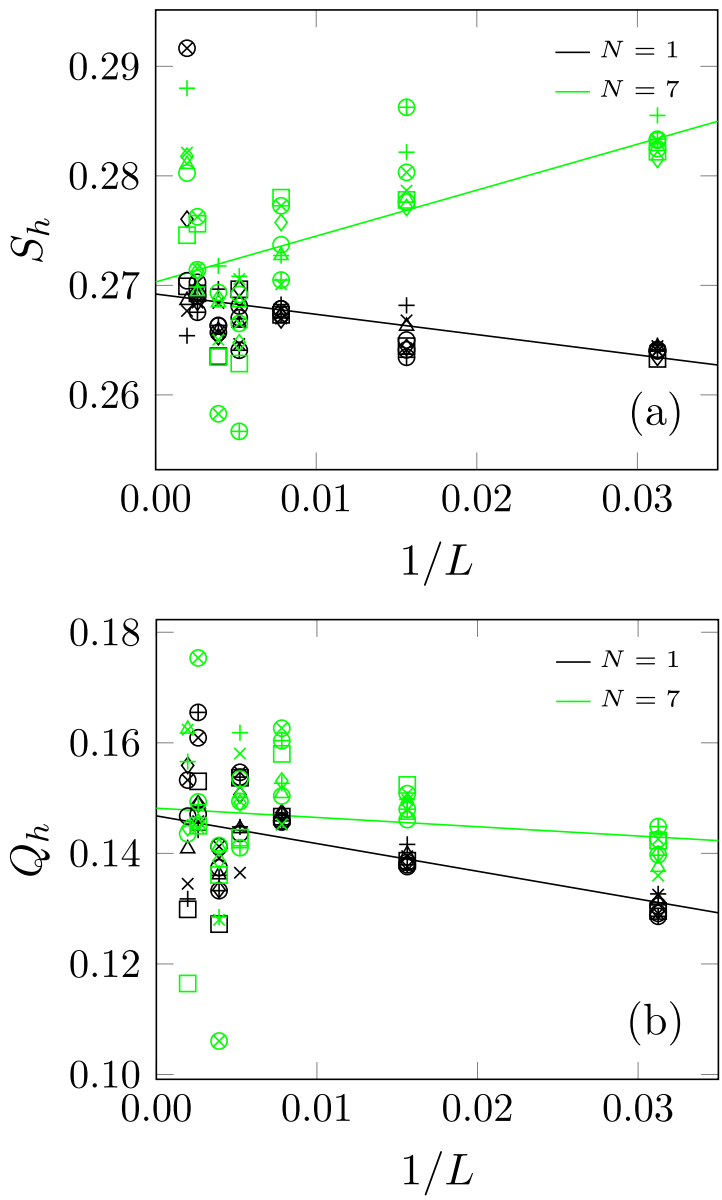

FIG. 4. Skewness $S_{h}$ (a) and kurtosis $Q_{h}$ (b) of the height distribution in the steady state plotted over the inverse lateral system size. Values are plotted only for $N=1$ (black) and $N=7$ (green) for the sake of clarity. The straight lines are linear fits, included to guide the eye. Different symbols indicate different ratios $t_{\text {start }} / t_{\text {steady* }} \geqslant 2$. A key is not provided for the symbols because there is no correlation with this parameter.

standard measures of the shape, the skewness

$$
S_{L}\left[\varphi_{L}\right]=\left\langle\Phi_{L}^{3}\left[\varphi_{L}\right]\right\rangle /\left\langle\Phi_{L}^{2}\left[\varphi_{L}\right]\right\rangle^{3 / 2}
$$

and the kurtosis

$$
Q_{L}\left[\varphi_{L}\right]=\left\langle\Phi_{L}^{4}\left[\varphi_{L}\right]\right\rangle /\left\langle\Phi_{L}^{2}\left[\varphi_{L}\right]\right\rangle^{2}-3,
$$

in the steady state. These measures were shown to be universal in KPZ models [38,39].

The obtained values for the width distribution $P_{L}\left[W^{2}(L)\right]$ show no significant dependence on $N$ nor $L$, our best results are $S=1.70(1)$ and $Q=5.38(4)$, in good agreement with those of Ref. [22].

For the distribution of surface heights, a weak correlation with the system size can be observed in Fig. 4. Heights were averaged in the steady state starting at different times $t_{\text {start }}>t_{\text {steady* }}$ (indicated by different symbols in the figure), but no dependence can be observed. Our results $S_{h}=0.270(5)$ and $Q_{h}=0.15(1)$ are in agreement with the ranges given in Ref. [40] and especially with the values $S_{h}=0.26(1)$ and $Q_{h}=0.134(15)$ reported in references [21,41]. Thus
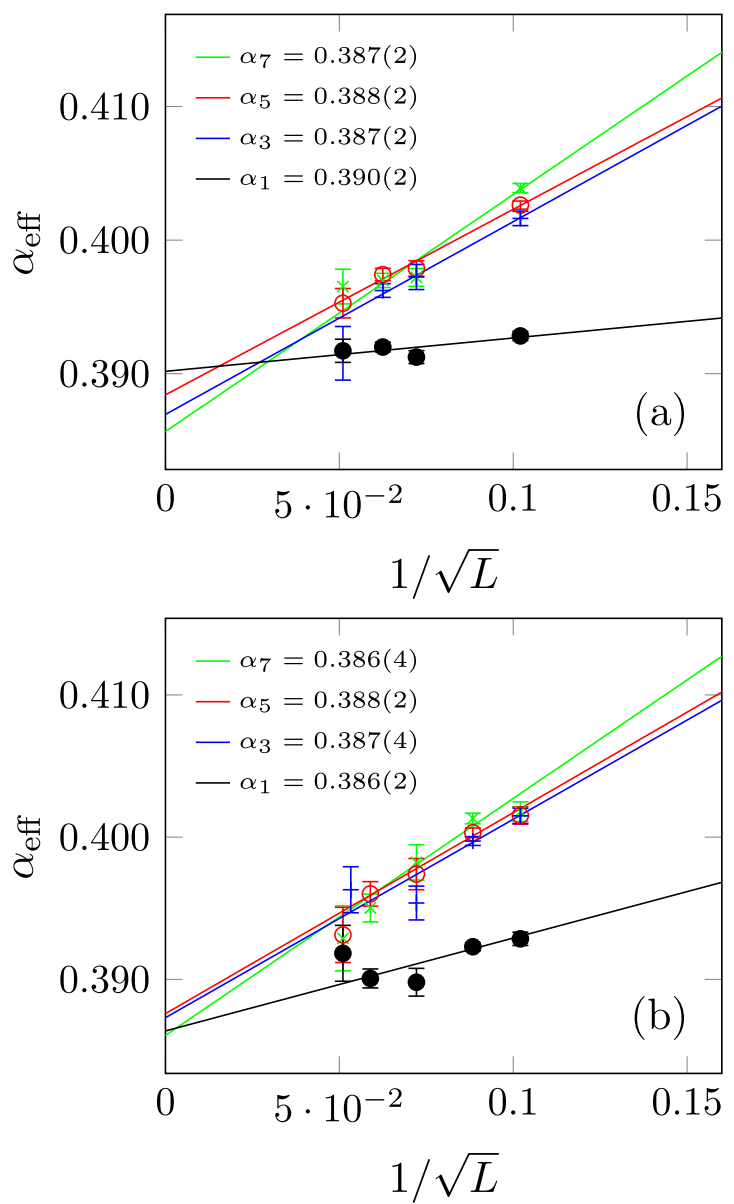

FIG. 5. Local slopes of finite-size scaling analysis for $N=$ $1,3,5,7$. Error bars are propagated $1 \sigma$ errors. Straight lines are linear fits to extrapolate to infinity, and uncertainties given for $\alpha_{N}$ are pure fit errors. Steady-state data are taken for $t>t_{\text {start }}=50 t_{\text {steady* }}$ (see text). (a) DD domains containing $6(+1) \times 10(+1)$ sites. Sample sizes are at least 512 realizations; for $N=5,7$ and sizes $L=64$ and 128 , $n=16384, n=8192$ are used. (b) DD domains containing $16 \times 16$ sites. For $L=512$ the sample contains 256 realizations; for other system sizes at least 512 samples are included.

all cumlant values are within error margins of the $\mathrm{KPZ}$ universality class irrespectively of $N$.

\section{Consistency of fine-size scaling with respect to DD}

Since we used a parallel DD in our simulations we have also checked for dependence of the results on the applied scheme. We performed additional finite-size scaling studies with domains of $16 \times 16$ and $6(+1) \times 10(+1)$ lattice sites. The figures in the parentheses refer to irregular tiling of the system. This is the consequence of the fact that lattices cannot be divided into domains with a lateral size of 6 (or 10) sites without remainder, thus a subset of domains have larger lateral size to compensate it. This configuration results from dividing the system into multiples of $5 \times 3$ tiles in order to achieve optimal load balancing on NVIDIA GTX Titan Black GPUs. In both cases the smallest considered system size was $L=64$ to avoid unreasonable DD. Another test was done using $3(+1) \times$ $5(+1)$-sized domains. These tiles turned out to be too small to 
give correct results, expressed by failing data collapses, and thus we do not consider them in the following discussion.

The differences among the results of the considered DD configurations were significant neither in the data collapses nor in the finite-size scaling fits. The most sensitive quantity proved to be the effective roughness exponent, shown in Fig. 5. Sample sizes of this test were smaller than those of Sec. III B, making the extrapolations less reliable. Still, all estimates derived from this data are consistent with the estimate $\alpha=0.390$ (4). Even the results of irregular, nonsquare DDs do not deviate significantly, although small systematic errors might be present.

\section{CONCLUSIONS}

Extensive numerical simulations have been performed for $(2+1)$-dimensional RSOS models with variable height difference restrictions. Careful correction to scaling analysis has provided numerical evidence that the universal surface growth exponents agree with the most precise values known for the $(2+1)$-dimensional KPZ class. These estimates, $\alpha=0.390(4)$ and $\beta=0.241(1)$, exclude the rational values $\alpha=4 / 10$ and $\beta=1 / 4$, conjectured by Refs. [25-27,31]. Our results support the generalized KPZ ansatz, which takes finite-time corrections into account and predicts exponents $x$ that are multiples of $\beta$ [37]. We found $x=0.90(2)$ for $N=1$ and $x \simeq 0.25$ for $N>1$.

We have shown that by increasing the local height differences we obtain better $S / N$ in the simulations, but stronger corrections to scaling, which can confuse numerical analysis based on simple power-law fitting. Therefore, smaller step-sized models, like the octahedron model [23], describe better the asymptotic, long-wave-scaling behavior of the KPZ universality class. Our conclusions for scaling corrections are in agreement with those obtained for ballistic growth models [24,32]. According to our knowledge oscillating convergence of effective exponents has not yet been observed in RSOS models, necessitating further investigations. We also provided estimates for the skewness $S=1.70(1)$ and the kurtosis $Q=5.38(4)$ of the surface width distributions as well as $S_{h}=0.270(5)$ and $Q_{h}=0.15(1)$ for the height distributions, both in the steady state. Our simulations have been performed using multisurface GPU SIMT algorithms with origin moving domain decomposition. The results have been justified by varying the tile sizes. A sustained performance of $\simeq 1.1 \times 10^{10}$ deposition attempts per second could be achieved running on a single NIVIDIA GTX Titan Black GPU. This opens up the possibility for precise RSOS simulations in higher dimensions.

\section{ACKNOWLEDGMENTS}

We thank S. Alves for sending us the correction-to-scaling plot of exponent $\alpha$ of the three-dimensional ballistic growth and S. C. Ferreira and T. Halpin-Healy for useful comments. Support from the Hungarian research fund OTKA (Grant No. K109577), the Initiative and Networking Fund of the Helmholtz-Gemeinschaft via the W2/W3 Programm (Grant No. W2/W3-026), and the International Helmholtz Research School NanoNet (Grant No. VH-KO-606) is acknowledged. We gratefully acknowledge computational resources provided by the HZDR computing center, NIIF Hungary, and the Center for Information Services and High Performance Computing (ZIH) at TU Dresden. We acknowledge support by the GCoE Dresden. J.K. thanks M. Weigel from Coventry University for providing a guest position, cofunded through the Erasmus+ program via the Leonardo-Büro Sachsen.
[1] M. Kardar, G. Parisi, and Y.-C. Zhang, Phys. Rev. Lett. 56, 889 (1986).

[2] T. Halpin-Healy, Phys. Rev. A 42, 711 (1990).

[3] J. M. Burgers, The Nonlinear Diffusion Equation: Asymptotic Solutions and Statistical Problems (Dordrecht-Holland, Boston, 1974).

[4] D. Forster, D. R. Nelson, and M. J. Stephen, Phys. Rev. A 16, 732 (1977).

[5] M. Kardar, Phys. Rev. Lett. 55, 2923 (1985).

[6] H. van Beijeren, R. Kutner, and H. Spohn, Phys. Rev. Lett. 54, 2026 (1985).

[7] H. Janssen and B. Schmittmann, Z. Phys. B 63, 517 (1986).

[8] T. Hwa, Phys. Rev. Lett. 69, 1552 (1992).

[9] P. Meakin, P. Ramanlal, L. M. Sander, and R. C. Ball, Phys. Rev. A 34, 5091 (1986).

[10] A. Barabási and H. Stanley, Fractal Concepts in Surface Growth (Cambridge University Press, Cambridge, 1995).

[11] J. Krug, Adv. Phys. 46, 139 (1997).

[12] M. Kardar, Nucl. Phys. B 290, 582 (1987).

[13] T. Kloss, L. Canet, and N. Wschebor, Phys. Rev. E 86, 051124 (2012).

[14] M. Schwartz and S. F. Edwards, Europhys. Lett. 20, 301 (1992).

[15] E. Frey and U. C. Täuber, Phys. Rev. E 50, 1024 (1994).
[16] M. Lässig, Nucl. Phys. B 448, 559 (1995).

[17] H. C. Fogedby, Phys. Rev. Lett. 94, 195702 (2005).

[18] L. Canet, H. Chaté, B. Delamotte, and N. Wschebor, Phys. Rev. E 84, 061128 (2011).

[19] B. M. Forrest and L.-H. Tang, Phys. Rev. Lett. 64, 1405 (1990).

[20] T. Halpin-Healy, Phys. Rev. Lett. 109, 170602 (2012).

[21] E. Marinari, A. Pagnani, and G. Parisi, J. Phys. A 33, 8181 (2000).

[22] F. D. A. Aarão Reis, Phys. Rev. E 72, 032601 (2005).

[23] J. Kelling and G. Ódor, Phys. Rev. E 84, 061150 (2011).

[24] S. G. Alves, T. J. Oliveira, and S. C. Ferreira, Phys. Rev. E 90, 052405 (2014).

[25] M. Lässig, Phys. Rev. Lett. 80, 2366 (1998).

[26] J. M. Kim and J. M. Kosterlitz, Phys. Rev. Lett. 62, 2289 (1989).

[27] J. M. Kim, J. M. Kosterlitz, and T. Ala-Nissila, J. Phys. A 24, 5569 (1991).

[28] T. Halpin-Healy, Phys. Rev. E 88, 042118 (2013).

[29] S. G. Alves, T. J. Oliveira, and S. C. Ferreira, Phys. Rev. E 90, 020103 (2014).

[30] A. Pagnani and G. Parisi, Phys. Rev. E 92, 010101 (2015).

[31] J. M. Kim, J. Korean Phys. Soc. 67, 1529 (2015).

[32] S. G. Alves and S. C. Ferreira, Phys. Rev. E 93, 052131 (2016).

[33] S. Alves (private communication). 
[34] J. Kelling, G. Ódor, M. F. Nagy, H. Schulz, and K. Heinig, Eur. Phys. J.: Spec. Top. 210, 175 (2012).

[35] J. Kelling, M. Weigel, G. Ódor, and S. Gemming (unpublished).

[36] G. Ódor, Universality in Nonequilibrium Lattice Systems (World Scientific, Singapore, 2008).

[37] T. J. Oliveira, S. G. Alves, and S. C. Ferreira, Phys. Rev. E 87, 040102 (2013).
[38] E. Marinari, A. Pagnani, G. Parisi, and Z. Rácz, Phys. Rev. E 65, 026136 (2002).

[39] G. Foltin, K. Oerding, Z. Rácz, R. L. Workman, and R. K. P. Zia, Phys. Rev. E 50, R639 (1994).

[40] T. Paiva and F. Aarão Reis, Surf. Sci. 601, 419 (2007).

[41] F. D. A. Aarão Reis, Phys. Rev. E 69, 021610 (2004). 